\title{
EVALUACIÓN PRELIMINAR DEL ESTADO DE CONSERVACIÓN DE PLANTAS: COMPARACIÓN DE DOS MÉTODOS APLICADOS AL GÉNERo GaVILEA (OrChIDACEAE)
}

\author{
PRELIMINARY EVALUATION OF THE CONSERVATION STATUS OF PLANTS: \\ COMPARISON OF TWO METHODS APPLIED TO THE GENUS GAVILEA (ORCHIDACEAE)
}

\author{
Victorio J. Dieta ${ }^{1}$ y Fernando Biganzoli* (D)
}

1. Instituto Nacional de Tecnología Agropecuaria (INTA). EEA Delta, AER Delta Frontal. Calle Las Casuarinas s/n. Tigre, Provincia de Buenos Aires, Argentina.

2. Departamento de Métodos Cuantitativos y Sistemas de Información, Facultad de Agronomía, Universidad de Buenos Aires. Av. San Martín 4453 CABA, Argentina.

*biganzol@agro.uba.ar

\section{Citar este artículo}

DIETA, V. J. \& F. BIGANZOLI. 2019 Evaluación preliminar del estado de conservación de plantas: comparación de dos métodos aplicados al género Gavilea (Orchidaceae). Bol. Soc. Argent. Bot. 54: 381-393.

DOI: http://dx.doi. org/10.31055/1851.2372.v54. n3.25362

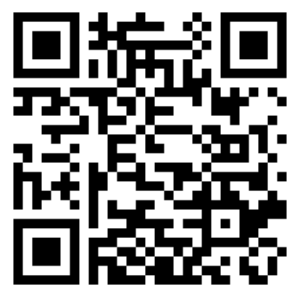

Recibido: 22 Febrero 2019 Aceptado: 26 Agosto 2019 Publicado: 30 Septiembre 2019 Editor: Omar Varela (D)

\section{SuMMARY}

Background and aims: It is essential to know the conservation status of the species, in order to prioritize intervention and management of the most threatened species populations. Usually available information is not enough for a definitive evaluation; therefore, it is important to develop rapid assessment methods of conservation status. In this work we performed a preliminary assessment of the conservation status for the Patagonian orchid genus Gavilea (Orchidaceae, Chloraeinae), and we compared our results with that obtained by experts.

M\&M: We compiled a database with location and collection date of herbarium specimens of the 17 species of Gavilea. We apply an adaptation of the criteria proposed by Krupnik et al., (2009) related to the geographical and temporal distribution of the collections. We compare the classification obtained with those published by experts.

Results: We categorize the 17 species, with 1 Potentially Extinct species, 6 Potentially Threatened species and the remaining 10 Potentially Not Threatened species. In addition, we found that 15 of the 17 species coincide in their classification with the evaluation of experts; only for G. platyantha, our categorization was more optimistic than that of experts.

Conclusions: The method allowed to categorize all the species and determine the priorities for future studies or interventions. In most cases, the categorization coincided with that carried out by experts. In general, we consider that the method is a good tool to generate an assessment of the preliminary conservation status of plant species.

\section{KEY WORDS}

Conservation status, expert evaluation, Gavilea, herbarium records, IUCN.

\section{RESUMEN}

Introducción y objetivos: Conocer el estado de conservación de las especies es fundamental para dar prioridad de intervención y manejo a las poblaciones de las especies más amenazadas. Muchas veces la información disponible no alcanza para una evaluación definitiva por lo que es importante desarrollar métodos de evaluación rápida. En este trabajo realizamos la evaluación preliminar del estado de conservación mediante información obtenida de ejemplares de herbario del género de orquídeas patagónicas Gavilea (Orchidaceae, Chloraeinae), y comparamos nuestros resultados con el estado de conservación de evaluaciones exhaustivas realizadas por expertos.

M\&M: Compilamos una base de datos con registros de localidad y fecha de colección de ejemplares de herbario de las 17 especies de Gavilea. Aplicamos una adaptación de los criterios propuestos por Krupnik y colaboradores (2009) relacionados con la distribución geográfica y temporal de las colecciones. Comparamos la clasificación obtenida con las publicadas por expertos.

Resultados: Categorizamos a las 17 especies, con 1 especie Potencialmente Extinta, 6 especies Potencialmente Amenazadas y las restantes 10 especies Potencialmente No Amenazadas. Encontramos que 15 de las 17 especies coinciden en su clasificación con la evaluación de expertos; solo para G. platyantha, nuestra categorización fue más optimista que la de expertos.

Conclusiones: El método permitió categorizar a todas las especies y determinar las prioridades para futuros estudios o intervenciones. En la mayoría de los casos, la categorización coincidió con la realizada por expertos. En general, consideramos que el método es una buena herramienta para generar una valoración del estado preliminar de conservación de las especies vegetales.

\section{Palabras clave}

Ejemplares de herbario, estado de conservación, Gavilea, evaluación de expertos, UICN. 


\section{INTRODUCCIÓN}

En la actualidad existe una rápida reducción de la biodiversidad que se debe, directa o indirectamente, a actividades humanas (Vitousek, 1992; Swingland, 2001; Van Dyke, 2008). Entre las principales causas de la disminución de biodiversidad se pueden mencionar la pérdida o alteración de hábitats, el cambio climático y la invasión de especies (Sala et al., 2000). Algunos indicadores de presión sobre la biodiversidad como el consumo de recursos por parte de los seres humanos, la fragmentación de hábitats y la presencia de especies exóticas invasoras han ido en aumento en las últimas cuatro décadas (Butchart et al., 2010). Ante esto, disminuir la tasa de pérdida de la biodiversidad en general, y en especial en plantas, es fundamental para satisfacer las necesidades presentes y futuras de bienes relacionados con los vegetales y mantener su papel fundamental como proveedoras de servicios ecosistémicos (Krupnick et al., 2009; Schatz, 2009). Conocer el estado de conservación de las especies, para saber cuáles son las especies amenazadas y así implementar herramientas de conservación adecuadas es de fundamental importancia (Rivers et al., 2010).

Anivel mundial, estimaciones basadas en especies endémicas indican que entre el 22 y el $47 \%$ de las especies de plantas se encontrarían amenazadas (Pitman \& Jorgensen, 2002). Sin embargo, solo se tienen datos del estado de conservación del $4 \%$ de las especies de plantas (Stuart et al., 2010, Rivers et al., 2010). La Estrategia Global para Conservación Vegetal (GSPC, por sus siglas en inglés) destaca la importancia de conocer el estado de conservación de las especies vegetales, afirmando la importancia de realizar evaluaciones preliminares del estado de conservación de todas las especies vegetales conocidas a nivel nacional, regional e internacional (UNEP, 2002). Sin embargo, el GSPC no especifica ningún método para realizar las evaluaciones preliminares del estado de conservación (Miller et al., 2012).

Existen distintos métodos para evaluar el estado de conservación de las plantas (Kricsfalusy \& Trevisan, 2014) que pueden agruparse en métodos basados en puntuación, en juicios cualitativos $\mathrm{y}$ en reglas de decisión (Mace et al., 2007). Los métodos basados en puntuación asignan valores a distinto número de atributos, y los combinan para obtener un índice del estado de conservación que serviría para ordenar a las especies consideradas. Un problema con estos métodos puntuables es que no existe un vínculo con el riesgo de extinción, y la ponderación de cada criterio es muchas veces arbitraria y frecuentemente obstaculizada por la falta de datos (Mace et al., 2007). Ejemplos de estos métodos son el índice que utilizaron Kricfalusy \& Trevisan (2014) para evaluar el estado de conservación de las especies raras de la flora de la provincia de Saskatchewan (Canadá) o el índice SUMIN propuesto por Reca et al. (1994) para evaluar el estado de conservación de la fauna. En este último índice, mediante la suma de los valores otorgados a 12 criterios se ordena a cada especie según prioridades de conservación. Algunas de las variables que utiliza son distribución de la especie, amplitud en el uso del hábitat, potencial reproductivo y tamaño poblacional (Acosta \& Murúa, 1999). El segundo conjunto de métodos evalúa el estado de conservación utilizando criterios cualitativos. La determinación del estado de una especie se realiza con base en la opinión de expertos (Master, 1991). Un ejemplo en la Argentina es el sistema PlanEAr (2009-2018), una base de datos de plantas endémicas de Argentina más algunas especies compartidas con Uruguay y con Chile. En este sistema se realiza una categorización preliminar del grado de amenaza de cada especie con base en información existente. Para la asignación de las categorías se privilegia el criterio de los expertos consultados. Las variables requeridas son área de distribución y abundancia relativa o rareza de la especie estudiada. Un problema de este tipo de métodos es la ponderación final tanto más incierta a medida que se evalúan especies con menos datos disponibles. El tercer conjunto de métodos incluye los basados en reglas de decisión, que utilizan criterios con límites o umbrales explícitos para asignar un riesgo de extinción. Este método es más objetivo para asignar categorías a las especies, necesitan relativamente menor cantidad de datos que las evaluaciones definitivas y brindan resultados repetibles y reevaluables frente a nueva información de las especies. El sistema más utilizado de este tercer tipo es la Lista Roja de la Unión Internacional para la Conservación de la Naturaleza (IUCN, por sus siglas en inglés), que ha sido ampliamente usado para completar las evaluaciones mundiales de conservación de 


\section{J. Dieta y F. Biganzoli - Evaluación preliminar del estado de conservación de plantas}

especies (Miller et al., 2013). La Lista Roja de la IUCN tiene como objetivo categorizar a las especies según su riesgo de extinción basándose en diferentes criterios (Rivers et al., 2010). Se puede evaluar el estado actual de una especie, su historia pasada, o se puede especular sobre su declive en el futuro (Miller et al., 2012). Algunos de los criterios utilizados por IUCN, como el A, C, D y E (IUCN, 2017) utilizan datos demográficos que son muy difíciles de conseguir en plantas, mientras que otros, como el criterio B, están asociados con la distribución geográfica de la especie (Miller et al., 2012).

Dentro del tercer grupo de métodos, encontramos también sistemas simplificados de evaluación preliminar del estado de conservación, que a partir de datos fácilmente disponibles podrían producir resultados verosímiles para un gran número de especies (Miller et al., 2012). Un ejemplo de estos sistemas simplificados es el desarrollado por el Jardín Botánico de Nueva York, que utiliza datos de especímenes de herbarios para identificar las especies que se encuentran "en riesgo" (Miller et al., 2013). Este método utiliza el cálculo de la extensión de las presencias (EOO, generalmente evaluada como el polígono convexo mínimo que incluye a todas las localidades conocidas de una especie) bajo el criterio B1 de la IUCN y clasifica a las especies como amenazadas si el área de distribución está por debajo de 20.000 $\mathrm{km}^{2}$ (IUCN, 2017). Otro método es el del Instituto Smithsoniano, desarrollado por Krupnick et al. (2009) que se basa en tres criterios relacionados con el área de distribución, con la cantidad de observaciones y con su distribución en el tiempo, utilizando como observaciones a ejemplares de herbarios.

La familia de las orquídeas presenta más especies amenazadas que cualquier otra familia de plantas (Nicholls 2004), y sus especies generalmente están entre los primeros organismos en desaparecer de un ecosistema perturbado (Dixon et al., 2003). Solamente un tercio de las especies de orquídeas son terrestres; sin embargo, casi la mitad de las especies extintas según IUCN de 1999, son plantas terrestres perennes y herbáceas (Swarts \& Dixon, 2009). Esto sugiere que las orquídeas terrestres estarían experimentando un mayor riesgo de extinción como resultado de múltiples factores de amenaza, como pérdida y degradación del hábitat, herbivoría o cambios en el régimen de disturbios (Swarts \& Dixon, 2009). El género Gavilea Poepp. está formado por diecisiete especies de orquídeas terrestres que habitan el sur de Argentina y el centro y sur de Chile (Zuloaga et al., 2008; Chemisquy, 2012). Varias especies crecen en áreas protegidas, pero también es común encontrarlas en áreas urbanas o periurbanas (Chemisquy, 2012). Los factores más importantes que ponen en riesgo sus poblaciones son el pastoreo, la urbanización, la introducción de animales como el jabalí, la invasión de plantas exóticas como la retama, el cambio del uso del suelo causado por la agricultura y la forestación, y los incendios (Lehnebach, 1999; Chemisquy, 2013). Chemisquy (2013), con base en el conocimiento de las especies y en la observación de muchas de sus poblaciones a campo, evaluó el estado de conservación de las especies argentinas y chilenas. Esta evaluación previa, complementada con evaluaciones para IUCN realizadas para las especies chilenas (Chamorro, 2008; Novoa, 2008; Tala, 2010) permiten analizar el desempeño de métodos rápidos de evaluación del estado de conservación basados en la información contenida en los ejemplares de herbario. En el presente trabajo utilizamos una adaptación del método de Krupnick et al. (2009) y comparamos los resultados con los obtenidos por expertos en el género Gavilea (Orchidaceae, Chloraeinae). Los resultados aquí obtenidos, podrían luego aplicarse a especies de plantas de las que no contamos con información detallada ni con el criterio de los expertos.

\section{Materiales y Métodos}

\section{Obtención de los datos}

Relevamos los datos de especímenes de Gavilea que se encuentran en los herbarios BAA, BAB y SI (sensu Thiers 2019), en la base de datos Documenta Florae Australis (www.darwin.edu. ar/iris) y citados en la bibliografía. De cada ejemplar obtuvimos los datos de localidad y fecha de colección. Eliminamos los ejemplares duplicados o con localidades inexistentes o que no pudimos interpretar. Confirmamos o completamos la georreferenciación de todos los ejemplares utilizando el programa Google Earth y cartografía de la zona. 


\section{Modelo de evaluación preliminar}

Para evaluar el estado de conservación de las especies de Gavilea adaptamos el método de Krupnick et al., (2009). Este método utiliza los criterios basados en la fecha, la distribución geográfica y la abundancia de las observaciones para clasificar a cada especie en tres categorías preliminares asociadas con las categorías de la Lista Roja de Especies de IUCN. Las tres categorías preliminares son: Potencialmente Extinto, que incluye las categorías extinto (EX) y extinto en estado silvestre (EW) de la Lista Roja de la IUCN,

Potencialmente Amenazada, que incluye las categorías en peligro crítico (CR), en peligro (EN) y vulnerable (VU), y Potencialmente No Amenazada, que incluye las categorías casi amenazada (NT) y preocupación menor (LC) de la Lista Roja de la IUCN (IUCN, 2017). Las adaptaciones que aplicamos al método se refieren a la forma de identificar subpoblaciones (Paso 2) y a los umbrales relacionados con características históricas en el área de estudio (Pasos 1, 3 y 4). A continuación, describimos los 4 pasos del método (Fig. 1) y las reglas de decisión utilizadas en este estudio:

Paso Uno: Nos permite evaluar si una especie disminuyó marcadamente la densidad de sus poblaciones o es extremadamente rara, hasta el punto de que ha sido indetectable por parte de los botánicos durante un largo período de tiempo hasta la actualidad. El año de corte debería ser el más alejado del presente que asegure una exploración suficiente del área o descripción del grupo en estudio. El año de corte que utilizamos es 1910, ya que en ese decenio encontramos un intenso esfuerzo de muestreo en Patagonia, principalmente como resultado de los viajes exploratorios de demarcación de límites internacionales (Del Vitto \& Petenatti, 1998). Las especies que solo registran colecciones antes de 1910 se considerarán potencialmente extintas.

Paso Dos: IUCN considera que si la especie posee seis o más subpoblaciones no estaría directamente amenazada por la desaparición estocástica de alguna subpoblación, mientras que si se encuentra en menos de seis subpoblaciones sería vulnerable (IUCN, 2017; Vulnerable, D2). Para la IUCN, la definición de subpoblaciones hace referencia a un área geográfica o ecológicamente distintiva en la cual un único evento podría afectar a todos los individuos de la especie (IUCN 2017). La forma de determinar el número de subpoblaciones de una especie plantea dificultades, particularmente si se realiza a partir de ejemplares de herbario. El método ideal debería ser independiente del número de colecciones, y debería describir a las especies y no a las colecciones. Rivers et al. (2010) proponen el método de círculos buffer ya que no muestra correlación entre número de subpoblaciones y de colecciones. En este método, cada localidad de un ejemplar de herbario se rodea con un círculo de un radio determinado, y los círculos superpuestos se fusionan, para formar una única subpoblación (Rivers et al., 2010). La longitud del radio utilizado debería basarse en datos de la biología de la especie analizada, en particular de la distancia máxima de dispersión de los propágulos. Hay registros de dispersión frecuente en orquídeas a distancias mayores a $40 \mathrm{~km}$. Por ejemplo, las orquídeas fueron unas de las primeras especies en colonizar las islas formadas luego de la erupción del volcán Krakatoa, que se encuentran a aproximadamente $40 \mathrm{~km}$ del continente (Partomihardjo, 2003). En otro estudio en los Países Bajos, la mediana de la dispersión a larga distancia de la orquídea Liparis loeselii (L.) Rich. fue de 50,6 km, con distancias observadas de hasta $220 \mathrm{~km}$ (Vanden Broeck et al., 2014). Con base en estos datos, el radio que utilizamos en este estudio fue de 50 kilómetros. Se utilizó para realizar el cálculo el software QGis (QGis 2.6.1). Además, a fin de determinar si el valor umbral arbitrario (aunque razonable) propuesto por la IUCN de seis subpoblaciones es determinante del resultado de la evaluación, repetimos el análisis utilizando los valores umbrales de 4 y 8 subpoblaciones.

Paso Tres: El número de colecciones sería un estimador indirecto de la abundancia de las poblaciones (Krupnick et al., 2009). Aunque esta relación podría estar afectada por otras causas como la densidad de rutas de acceso al área ocupada por la especie, el tamaño de la planta, el atractivo de sus flores o los usos, entre otros, puede hacerse el supuesto que una especie es colectada más frecuentemente si es abundante que si es escasa. Krupnick et al. (2009) proponen que las especies con menos colecciones que el valor mediano para la región en estudio se considerarán raras, mientras que las especies que tengan más colecciones se considerarán comunes. Para la región Patagónica, utilizamos una base de datos compilada específicamente para analizar especies de Patagonia 


\section{J. Dieta y F. Biganzoli - Evaluación preliminar del estado de conservación de plantas}

Evaluación

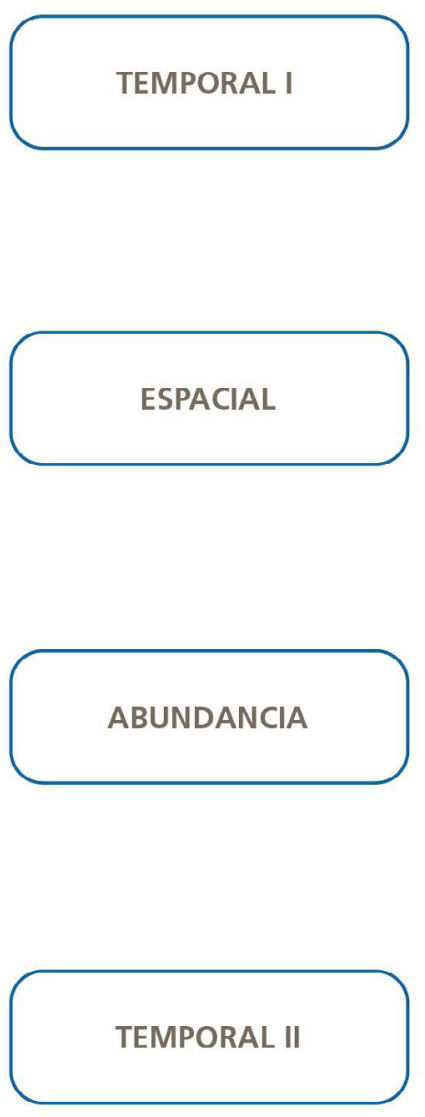

Pasos

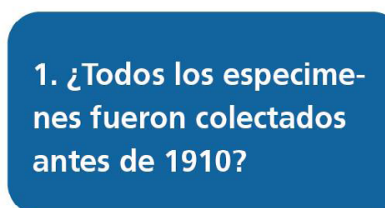$$
\sqrt{5}
$$

NO

Después de 1910
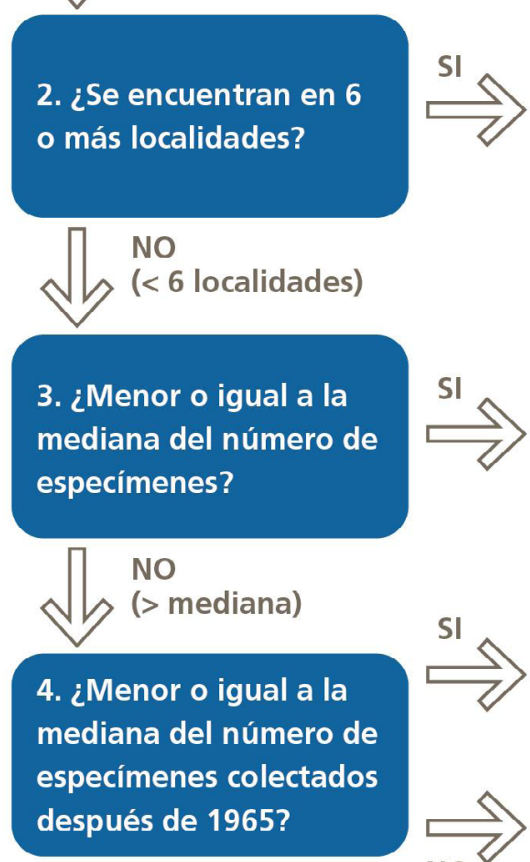

Resultados

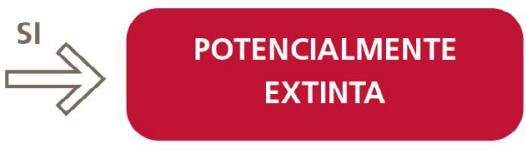

POTENCIALMENTE

NO AMENAZADA

(> mediana)

Fig. 1. Pasos del modelo de evaluación rápida, con base en criterios temporales, espaciales y de abundancia. Modificado de Krupnick et al., 2009.

(Biganzoli, datos no publicados). La base de datos cuenta con información de 670 especies, 23452 colecciones y fue utilizada previamente como control del esfuerzo de colección en Patagonia (Biganzoli et al., 2013). El número de colecciones registrado en la base de datos para cada especie en esta región varía ampliamente entre 1 (83 especies) y 485 ejemplares (1 especie), con un valor mediano de 13 ejemplares. Para separar poblaciones abundantes de escasas, comparamos el número de ejemplares colectados de cada especie de Gavilea, con la mediana general de plantas colectadas en Patagonia. Si la cantidad de colecciones de una especie de Gavilea fue menor a la mediana general, consideramos a la especie como rara y consecuentemente, potencialmente amenazada.

Paso Cuatro: Para evaluar la posible disminución de las poblaciones en el tiempo, utilizamos la frecuencia de colecciones en los últimos años. Las especies que pasaron el paso tres poseen un número relativamente grande de ejemplares, mayores a la mediana general. De estas especies, las que tengan relativamente pocas colecciones en los últimos años se considerarán actualmente raras o declinantes (Krupnick et al., 2009), mientras que las especies que tengan abundantes colecciones en los últimos 
años se considerarán comunes. El año de corte que utilizamos fue el año 1965, donde encontramos un período importante de colectas de plantas en Patagonia originadas principalmente por los importantes viajes relacionados con la publicación de Flora Patagónica del INTA (Del Vitto \& Petenatti, 1998). El valor de colecciones en los últimos años para separar especies declinantes de comunes lo obtuvimos de la base de datos de Patagonia utilizada en el paso 3. La mediana de ejemplares colectados de plantas en general en Patagonia desde 1965 hasta la actualidad fue de cinco ejemplares. Comparamos la cantidad de ejemplares colectados para cada especie de Gavilea desde 1965 hasta la actualidad con el valor general para Patagonia. Las especies con cinco o menos colecciones serían raras o declinantes $\mathrm{y}$, consecuentemente, Potencialmente amenazadas. Las especies de Gavilea con más de cinco ejemplares serían Potencialmente No Amenazadas. Las fechas y valores de abundancia utilizados como umbrales podrían afectar significativamente el resultado de la clasificación, por lo que evaluamos la sensibilidad de los resultados a estos valores umbrales. Reanalizamos los datos utilizando los años 1960 y 1970 y los valores de colecciones generales correspondientes a esos años para la evaluación.
Comparamos las categorías obtenidas mediante el modelo de evaluación preliminar con la evaluación de expertos realizada por Chemisquy (2013), Chamorro (2008), Novoa (2008) y Tala (2010), siguiendo el proceso de la Lista Roja de la IUCN. Estos autores evaluaron el estado de conservación de las especies de Gavilea con base en datos obtenidos en viajes de campo, bibliografía y revisión de ejemplares de herbarios. A partir de la comparación de datos analizamos las similitudes y diferencias encontradas para identificar los criterios responsables de las discrepancias entre métodos.

\section{Resultados}

Compilamos una base de datos con 622 ejemplares de Gavilea. La especie con menor número de ejemplares fue Gavilea kingii con uno, mientras que la especie con más colecciones fue Gavilea lutea con 159 (Fig. 2). La mediana del número de ejemplares por especie fue de catorce, muy cercano al valor general (13) obtenido para Patagonia.

Evaluación preliminar. En el primer paso de la evaluación, solo G. kingii, con un único ejemplar coleccionado en la localidad tipo hace más de 100

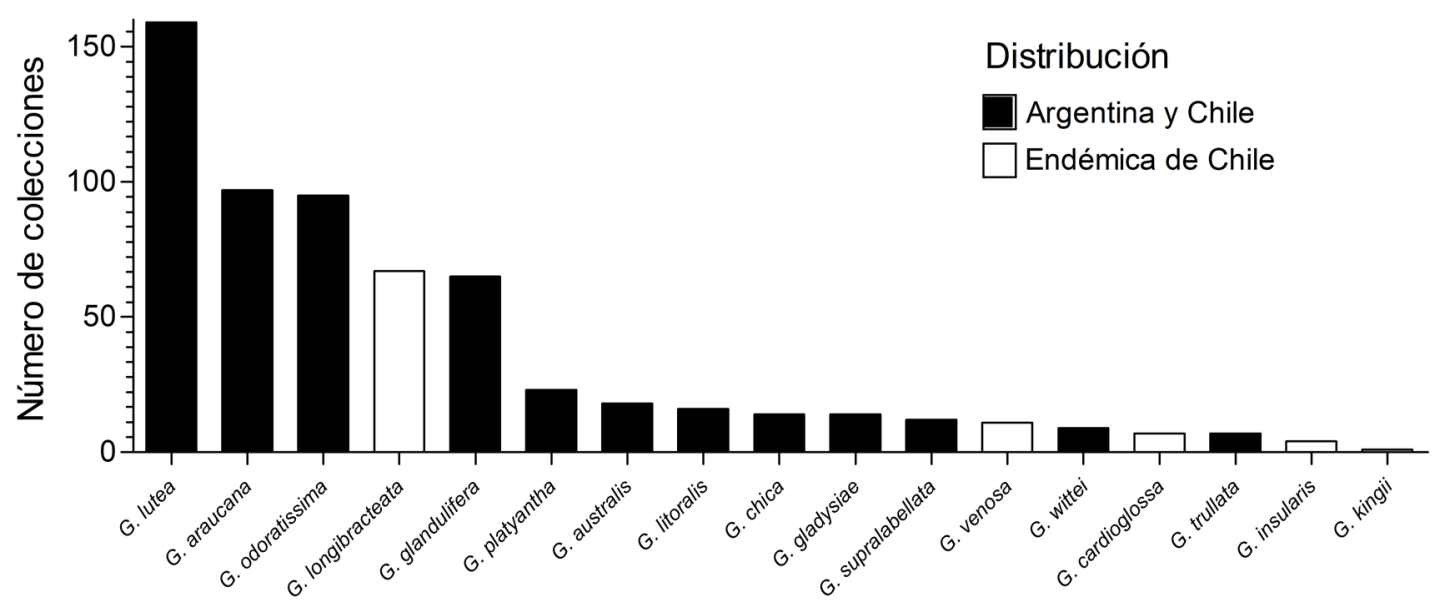

Especies

Fig. 2. Número de colecciones y distribución geográfica de las especies del género Gavilea. 


\section{J. Dieta y F. Biganzoli - Evaluación preliminar del estado de conservación de plantas}

años (en 1846), fue clasificada preliminarmente como potencialmente extinta (Fig. 3). En el segundo paso, encontramos que seis de dieciséis especies poseen más de seis subpoblaciones, y fueron clasificadas preliminarmente como Potencialmente No Amenazadas. El análisis de sensibilidad para este criterio utilizando valores umbrales de cuatro u ocho subpoblaciones, mostró que el criterio es robusto al valor umbral, con solo una diferencia al utilizar cuatro subpoblaciones. En este caso, G. wittei cambia de Potencialmente Amenazada a Potencialmente No Amenazada. En el tercer paso, seis de las diez especies con menos de seis subpoblaciones poseen menos de 13 colecciones, por lo que fueron clasificadas preliminarmente como Potencialmente Amenazadas. En el cuarto paso encontramos que las cuatro especies evaluadas tienen una alta proporción de las colecciones en los últimos 50 años por lo que preliminarmente las consideramos como Potencialmente No Amenazadas. A pesar de la contrastante cantidad de colecciones entre G. gladysiae y G. australis con relación a $G$. lutea y $G$. longibracteata (Fig. 4), las 4 especies tienen al menos el doble de colecciones que el valor umbral de 5 colecciones para este criterio. Esta clasificación no cambió cuando consideramos otros años de corte, esto es 1960 o 1970 (con sus respectivos valores umbrales de 7 y 4 colecciones). En resumen, con nuestra evaluación preliminar clasificamos a seis especies del género Gavilea como potencialmente amenazadas, y una como potencialmente extinta (Fig. 3).

Comparación con la evaluación de expertos, basados en criterios de IUCN: Encontramos que con el método rápido obtuvimos casi la misma clasificación que en las evaluaciones publicadas por expertos y solo para tres especies la clasificación fue diferente (Tabla 1). La primera de ellas fue G. venosa,

\section{Resultados del modelo de Evaluación}

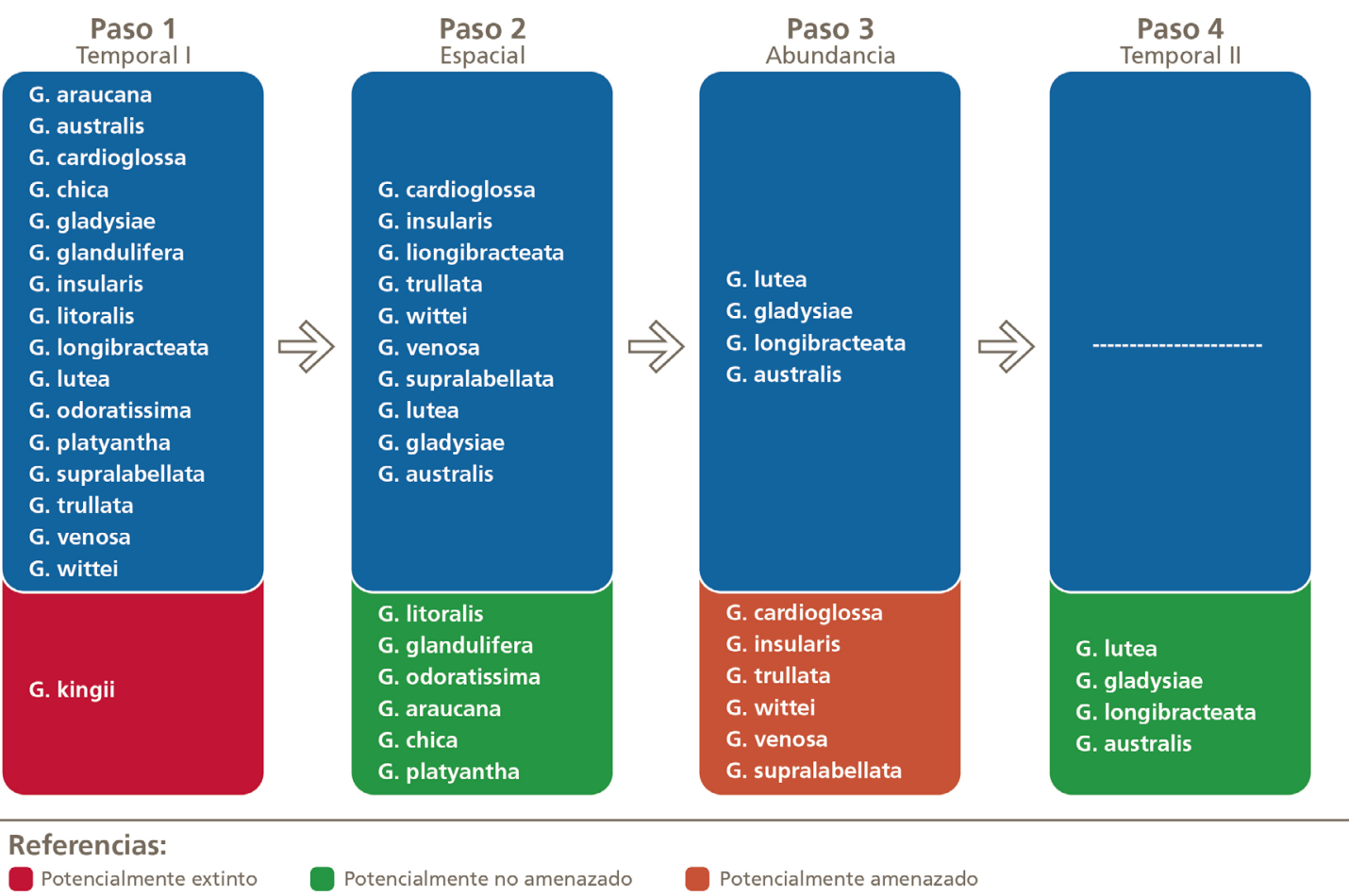

Fig. 3. Categorización de las especies de Gavilea, con base en el modelo de evaluación rápida. 

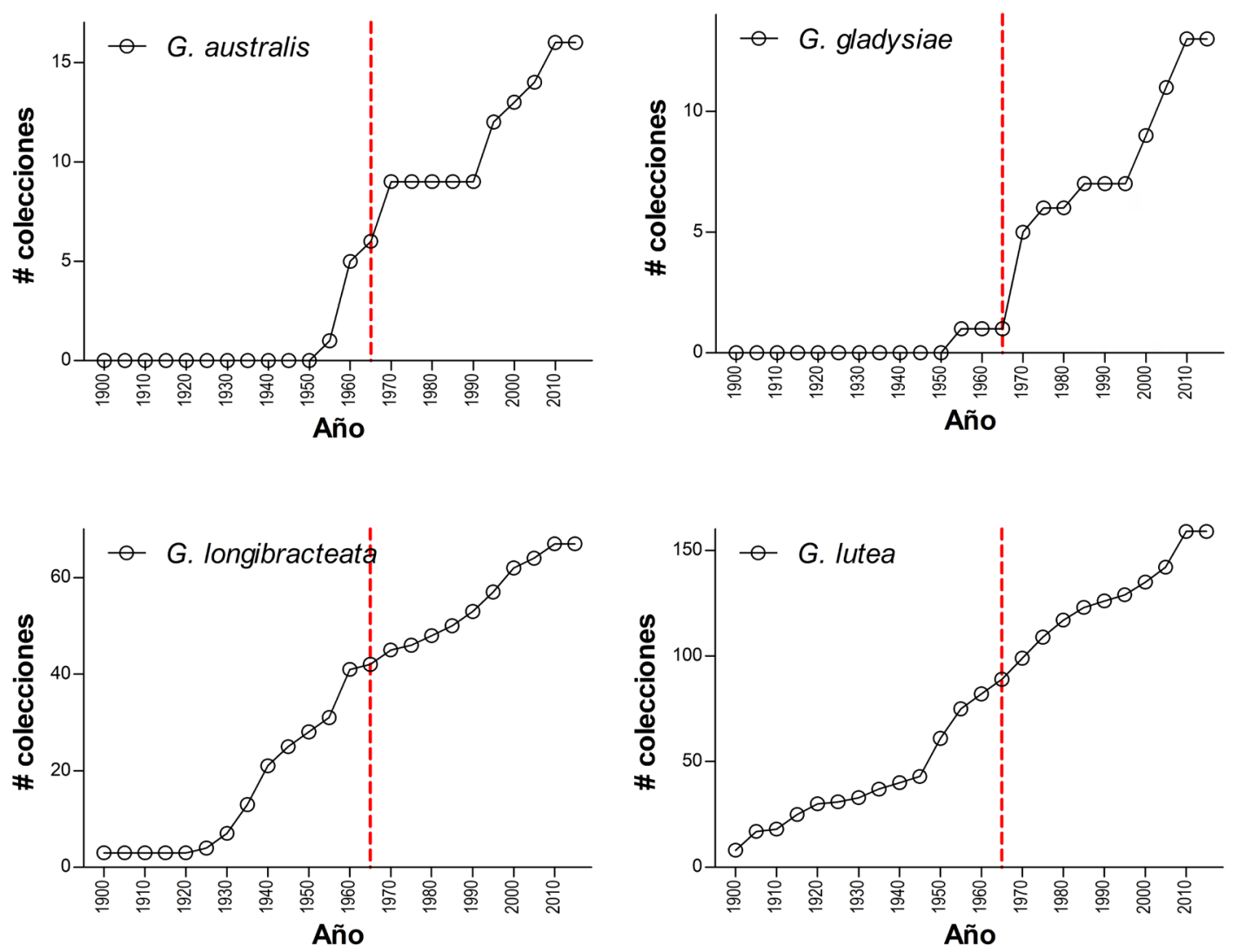

Fig. 4. Número de colecciones acumuladas cada 5 años de las especies de Gavilea categorizadas como "Potencialmente No Amenazadas" en el paso cuatro de la evaluación rápida.

Tabla 1. Comparación de la evaluación rápida realizada en este trabajo con las evaluaciones publicadas por expertos: entre paréntesis, comparación con Chamorro (2008) y Tala (2010); sin paréntesis,

\section{comparación con Chemisquy (2013).}

\begin{tabular}{|c|c|c|c|c|}
\hline & & & luación rápida & \\
\hline & & Extinta & Amenazada & No Amenazada \\
\hline & Extinta & G. kingii & & \\
\hline & & & G. supralabellata & \\
\hline & & & G. trullata & \\
\hline & Amenazada & (G. kingii) & G. witeii & G. platyantha \\
\hline sू & & & G. insularis (G. insularis) & \\
\hline ț & & & G. cardioglossa & \\
\hline$\frac{1}{0}$ & & & & G. longibracteata \\
\hline - & & & & G. glandulifera \\
\hline ] & & & & G. odoratissima \\
\hline$\frac{\sqrt{2}}{2}$ & & & & G. araucana \\
\hline & No & & G. venosa & G. lutea \\
\hline & Amenazada & & & G. chica \\
\hline & & & & G. gladysiae \\
\hline & & & & G. australis \\
\hline & & & & G. litoralis \\
\hline
\end{tabular}




\section{J. Dieta y F. Biganzoli - Evaluación preliminar del estado de conservación de plantas}

que clasificamos como Potencialmente Amenazada porque posee menos de seis subpoblaciones, y además la cantidad de ejemplares coleccionados que posee es menor al valor umbral que utilizamos para determinar rareza. Chemisquy (2013), en cambio, la clasifica como casi amenazada (NT), basándose en su distribución espacial. La segunda es $G$. platyantha, que preliminarmente clasificamos como Potencialmente No Amenazada, porque tiene más de seis subpoblaciones. Chemisquy (2013), en cambio, la clasifica como vulnerable (VU) debido a los pocos ejemplares que existen en los herbarios. La tercera es una de las tres especies de Gavilea endémicas de Chile; Tala (2010) clasificó a $G$. kingii en peligro (EN), en cambio nosotros la clasificamos como potencialmente extinta. En este caso, las evaluaciones difieren en los ejemplares utilizados para realizarlas. Por otra parte, Novoa (2008) no pudo clasificar a $G$. cardioglossa por tener datos insuficientes (DD).

\section{Discusión}

Nuestros resultados mostraron que solo una de las diecisiete especies estaría Potencialmente Extinta, y seis especies estarían Potencialmente Amenazadas. Sobre estas especies es donde deberían centrarse los estudios posteriores sobre conservación, por ejemplo estudios ecológicos, esfuerzos en viajes de exploración en busca de poblaciones, y evaluación de expertos. Las diez especies restantes no estarían amenazadas, por lo que estudios futuros no serían una prioridad. Gavilea kingii es la única especie de Gavilea que clasificamos como Potencialmente Extinta. Esta especie tiene un único ejemplar coleccionado, hace más de 150 años, es de extrema rareza, y sería muy importante en este caso nuevos estudios a campo en su zona de distribución. Todas las especies potencialmente amenazadas de Gavilea se relacionan con el paso 3 de la evaluación. Estas especies tienen menos de 6 subpoblaciones y escasas colecciones, posiblemente relacionado con baja abundancia en la naturaleza. Esta combinación de factores las haría más vulnerables a cambios en el uso del suelo, a procesos estocásticos o al cambio climático (Lomolino et al., 2016; Hoffmann et al., 2017). Los principales cambios en el uso del suelo de la región son el aumento de la urbanización, con superficies ocupadas por viviendas y caminos, afectando en particular a aquellas especies que crecen en los alrededores de zonas urbanas (Chemisquy, 2013), el reemplazo y fragmentación del bosque nativo, por desarrollos forestales y expansión de áreas de pasturas (Rodríguez-Echeverry et al., 2015), que además de modificar el hábitat de las orquídeas intensifica el ramoneo de los animales sobre las hojas carnosas de las orquídeas, sobre todo en los límites de la estepa y los bosques patagónicos (Lehnebach, 1999). También algunas especies podrían verse afectadas por los efectos del cambio global, a través del aumento de la temperatura media (IPCC 2007), sobre todo aquellas especies como Gavilea australis, que poseen la distribución más austral, a las que se les restringiría su hábitat, o directamente desaparecería (Hickling et al., 2006; Swarts \& Dixon, 2009). A pesar de que la declinación de las poblaciones es un criterio clave para la IUCN (criterios B y C, IUCN, 2017) y es una etapa intermedia antes de la extinción de una especie (Collen et al., 2011), ninguna de las especies evaluadas hasta el paso 4 , fue clasificada como potencialmente amenazada debido a que disminuyó la frecuencia de colecciones. Esto podría deberse a que solo llegaron a este paso especies relativamente abundantes, a que las orquídeas son atractivas para los botánicos que las encuentran y herborizan aunque éstas no sean frecuentes en la naturaleza (McCarthy, 1998; Guralnick \& Van Cleve, 2005; Stropp et al., 2016) o a que existe un retraso en la detección de tendencias a través de las colecciones depositadas en los herbarios (James et al., 2018).

El desempeño del método adaptado de Krupnic et al., (2009) fue muy bueno comparado con las categorizaciones de los expertos (Chamorro, 2008; Novoa, 2008; Tala, 2010; Chemisquy, 2013) ya que llegamos a la misma clasificación en quince de las diecisiete especies. Solo dos especies, G. venosa y $G$. platyantha, tienen una clasificación diferente. G. venosa es una especie endémica de Chile. La clasificamos como potencialmente amenazada, debido a que los 11 ejemplares coleccionados de esta especie están agrupados en tres subpoblaciones, número menor al umbral en el paso dos, y a que el número de ejemplares coleccionados es menor a la mediana general en el paso tres. Chemisquy (2013) la clasifica como casi amenazada (NT), mencionando que posee una distribución más 
restringida que otras especies de Gavilea. En este caso, las categorías asignadas son muy similares, y nuestra clasificación, algo más "pesimista" no sería problema bajo el principio de precaución (Burgman, 2005). En cuanto a $G$. platyantha, la evaluación de expertos (Chemisquy, 2013) la considera como amenazada, en la categoría Vulnerable (VU) de la IUCN, debido a que posee un pequeño número de ejemplares, más allá de su amplia distribución. En nuestra clasificación, $G$. platyantha fue clasificada como No Amenazada ya que las colecciones superan la mediana general y están agrupadas en 8 subpoblaciones. La discrepancia en este caso está dada por la importancia que el método otorga a la separación en subpoblaciones de las localidades registradas. Es por esto que la determinación de las subpoblaciones y la distancia entre ellas no es trivial y es dependiente del grupo en estudio (Rivers et al., 2010). Una tercera discrepancia aparece con la categorización realizada por Tala (2010) de Gavilea kingii. En este caso las diferencias fueron causadas por la utilización de ejemplares incorrectamente determinados; Chemisquy (2012) comprobó que solo es válido el único ejemplar coleccionado hace más de 100 años. Esto resalta la importancia de revisar las determinaciones o, en su defecto, incluir ejemplares determinados solo por especialistas confiables. Estudios previos han mostrado que incluso unos pocos ejemplares con determinaciones incorrectas pueden llevar a decisiones contrastantes (Vogel Ely et al., 2017; Bried et al., 2018). Además, hay que considerar la escala geográfica de las categorizaciones (Gärdenfors et al., 2001; Helfman, 2013). Por ejemplo, Vidal et al. (2012) analizaron las orquídeas del Parque Nacional Torres del Paine (Chile) y, basándose en el criterio de abundancia, realizaron la categorización de las cinco especies de Gavilea que se encuentran en el Parque. Si consideramos que las especies comunes no estarían amenazadas, para tres de las 5 especies analizadas las evaluaciones propondrían categorizaciones diferentes. Gavilea gladysiae fue clasificada como muy rara y Gavilea litoralis como rara en la escala del Parque, mientras que a escala global no estarían amenazadas; Gavilea supralabellata fue categorizada como común dentro del Parque, mientras que a escala global la clasificamos como Potencialmente Amenazada. Esto resalta la importancia de la escala geográfica al realizar las evaluaciones del estado de conservación.

\section{Conclusiones}

El resultado de nuestra categorización se asemeja a los resultados obtenidos por la evaluación de expertos, usando los criterios de IUCN. Esto es bastante esperable ya que los datos primarios utilizados fueron similares o parcialmente superpuestos en muchos casos. Sin embargo, es promisorio que se pueda llegar a un orden de prioridad para investigaciones futuras sin el trabajo de expertos para los grupos taxonómicos o áreas geográficas donde no existen. Posteriormente, para una evaluación definitiva y para definir acciones de conservación, el trabajo de taxónomos, ecólogos y manejadores expertos sería imprescindible. Comparaciones recientes del método original de Krupnik y colaboradores con otros métodos (Nic Lughadha et al., 2018) resaltó la influencia que tiene en las categorizaciones el criterio del número de subpoblaciones. Esto hace que sea extremadamente importante una correcta georreferencación de las localidades de colección y una determinación biológicamente informada de la distancia entre subpoblaciones para el grupo de especies evaluadas. El desarrollo de modelos de estado de conservación basados en datos de herbarios tiene un costo relativamente bajo, en comparación con estudios de campo, para determinar el grado de amenaza de las especies, y un punto de partida para el desarrollo de políticas de conservación de las especies, que luego tendrán que ser refrendadas a través de la evaluación de expertos. Una estimación reciente mostró que solo el 32,9\% de las colecciones de 13 grandes herbarios del mundo se encuentran disponibles en línea (Holmes et al., 2016). Este número disminuye ampliamente si consideramos instituciones pequeñas que pueden mantener colecciones localmente importantes para regiones subrepresentadas en los grandes herbarios. Es fundamental entonces, para poder lograr evaluaciones rápidas, aumentar las colecciones depositadas en herbarios, digitalizar los datos que se encuentran en ellos y aumentar su disponibilidad.

\section{Contribución de los Autores}

VD y FB diseñaron la investigación, compilaron la base de datos y participaron en la escritura del manuscrito, VD analizó los datos. 


\section{J. Dieta y F. Biganzoli - Evaluación preliminar del estado de conservación de plantas}

\section{Agradecimientos}

Al personal de los herbarios FAA, BAB y SI por su colaboración durante la revisión de material. A C. Godoy-Bürki y P. Graff por sus comentarios en versiones previas de este manuscrito y a M. F. Dieta por la ayuda con la edición de las figuras.

\section{Bibliografía}

ACOSTA, J. C. \& F. MURÚA. 1999. Lista preliminar y estado de conservación de la mastofauna del Parque Natural Ischigualasto, San Juan, Argentina. Multequina 8: 121-129.

BIGANZOLI, F., C. LARSEN \& A. G. ROLHAUSER. 2013. Range expansion and potential distribution of an invasive grass (Bromus tectorum) in southern South America on the base of herbarium records. Journal of Arid Environments 97: 230236.

http://dx.doi.org/10.1016/j.jaridenv.2013.07.006

BRIED, J. T., B. E. ALLENA, E. T. AZERIAA, V. E. CRISFIELDB \& M. J. WILSON. 2018. Experts and models can agree on species sensitivity values for conservation assessments. Biol. Conservation 225: 222-228.

http://dx.doi.org/10.1016/j.biocon.2018.07.013

BURGMAN, M. 2005. Risks and Decisions for Conservation and Environmental Management. Cambridge University Press, Cambridge, UK.

BUTCHART, S. H. M., M. WALPOLE, B. COLLEN, A. VAN STRIEN, J. P. W. SCHARLEMANN, R. E. A. ALMOND, J. E. M. BAILLIE, B. BOMHARD, C. BROWN, J. BRUNO, K. E. CARPENTER, G. M. CARR, J. CHANSON, A. M. CHENERY, J. CSIRKE, N. C. DAVIDSON, F. DENTENER, M. FOSTER, A. GALLI, J. N. GALLOWAY, P. GENOVESI, R. D. GREGORY, M. HOCKINGS, V. KAPOS, J. F. LAMARQUE, F. LEVERINGTON, J. LOH, M. A. MCGEOCH, L. MCRAE, A. MINASYAN, M. HERNÁNDEZ MORCILLO, T. E. E. OLDFIELD, D. PAULY, S. QUADER, C. REVENGA, J. R. SAUER, B. SKOLNIK, D. SPEAR, D. STANWELL-SMITH, S. N. STUART, A. SYMES, M. TIERNEY, T. D. TYRELL, J. C. VIÉ \& R. WATSON. 2010. Global Biodiversity: Indicators of Recent Declines. Science 328: 11641168. http://dx.doi.org/10.1126/science. 1187512
COLLEN, B., L. MCRAE, S. DEINET, A. DE PALMA, T. CARRANZA, N. COOPER, J. LOH \& J. E. M. BAILLIE. 2011. Predicting how populations decline to extinction. Phil. Trans. R. Soc. B 366: 2577-2586. http://dx.doi.org/10.1098/rstb.2011.0015

CHAMORRO, M. R. 2008. Gavilea insularis. [online]. Disponible en: www.mma.gob.cl/ clasificacionespecies/ informacion-procesos-2014. htm [Acceso: 20 enero 2019].

CHEMISQUY, M. A. 2012. Revisión taxonómica del género Gavilea (Ochidaceae, Chloraeinae). Darwiniana 50: 33-80.

http://dx.doi.org/10.14522/darwiniana.2014.501.340

CHEMISQUY, M. A. 2013. Evaluación del estado de conservación de las especies del género Gavilea (Orchidaceae, Chloraeinae) en Argentina y Chile. Revista Mus. Argent. Ci. Nat., N. S. 15: 161-167.

DEL VITTO, L. A. \& E. M. PETENATTI. 1998. Evolución del conocimiento botánico de la Patagonia Argentina. En: M. N. Correa (Ed.). Flora Patagónica, Tomo VIII, Parte I. Instituto Nacional de Tecnología Agropecuaria, Buenos Aires, pp 167-265.

DIXON, K. W., P. J. CRIBB, S. P. KELL \& R. L. BARRETT (Eds.). 2003. Orchid conservation. Kota Kinabalu, Sabah: Natural History Publications.

GÄRDENFORS, U., C. Hilton-Taylor, G. M. Mace \& J. P. Rodríguez. 2001. The Application of IUCN Red List Criteria at Regional Levels. Conservation Biol. 15: 1206-1212. http://dx.doi.org/10.1111/j.1523-1739.2001.00112.x

GURALNICK, R. \& J. VAN CLEVE. 2005. Strengths and weaknesses of museum and national survey data sets for predicting regional species richness: comparative and combined approaches. Diversity \& Distrib. 11: 349-359. http://dx.doi.org/10.1111/j.1366-9516.2005.00164.x

HELFMAN, G. S. 2013. National "versus" global red lists of imperiled fishes: why the discord? Environ. Biol. Fish 96:1159-1168.

http://dx.doi.org/10.1007/s10641-011-9843-x

HICKLING, R., D. B. ROY, J. K. HILL, R. FOX \& C. D. THOMAS. 2006. The distributions of a wide range of taxonomic groups are expanding polewards. Global Change Biol. 12: 450-455.

http://dx.doi.org/10.1111/j.1365-2486.2006.01116.x

HOFFMANN, A. A., CARLA. M. SGRÒ \& T. N. KRISTENSEN. 2017. Revisiting adaptive potential, population size, and conservation. Trends Ecol. Evol. 32: 506-517.

http://dx.doi.org/10.1016/j.tree.2017.03.012 
HOLMES, M. W., T. T. HAMMOND, G. O. U. WOGAN, R. E. WALSH, K. LABARBERA, E. A. WOMMACK, F. M. MARTINS, J. C. CRAWFORD, K. L. MACK, L. M. BLOCH \& M. W. NACHMAN. 2016. Natural history collections as windows on evolutionary processes. Molec. Ecol. 25:864-881. http://dx.doi.org/10.1111/mec.13529

IPCC. 2007. Climate change 2007: the physical science basis. Contribution of Working Group I to the Fourth Assessments Report of the Intergovernmental Panel on Climate Change. In: SOLOMAN, S., D. QIN, M. MANNING, Z. CHEN, M. MARQUIS, K. B. AVERYT, M. TIGNOR \& H. L. MILLER (eds.). Cambridge University Press, United Kingdom and New York, USA.

IUCN STANDARDS AND PETITIONS SUBCOMMITTEE. 2017. Guidelines for using the IUCN Red List categories and criteria. Version 13. www.iucnredlist.org/documents/RedListGuidelines.pdf

JAMES, S. A., P. S. SOLTIS, L. BELBIN, A. D. CHAPMAN, G. NELSON, D. L. PAUL \& M. COLLINS. 2018. Herbarium data: Global biodiversity and societal botanical needs for novel research. Appl. Plant Sciences 6: e1024. http://dx.doi.org/10.1002/aps3.1024

KRICSFALUSY, V. V. \& N. TREVISAN. 2014. Prioritizing regionally rare plant species for conservation using herbarium data. Biodivers. \& Conservation 23: 39-61.

http://dx.doi.org/ 10.1007/s10531-013-0583-4

KRUPNICK, G. A., W. J. KRESS \& W. L. WAGNER. 2009. Achieving target 2 of the global strategy for plant conservation: building a preliminary assessment of vascular plant species using data from herbarium specimens. Biodivers. \& Conservation 18: $1459-1474$.

http://dx.doi.org/10.1007/s10531-008-9494-1

LEHNEBACH, C. A. 1999. Current status of the Chilean orchid flora. Orchid Conservation News 2: 8-10.

LOMOLINO, M. V.; B. R. RIDDLE \& R. J. WHITTAKER. 2016. Biogeography. Fifth Edition. Sinauer Associates, Oxford University Press.

MACE, G. M., H. P. POSSINGHAM \& N. LEADERWILLIAMS. 2007. Prioritizing choices in conservation. En: Macdonald, D. \& K. Service (Eds.). Key topics in conservation biology. Blackwell Publishing, Malden, pp 17-34.

MASTER, L. L. 1991. Assessing threats and setting priorities for conservation. Conservation Biol. 5: 559-563. http:// dx.doi.org/10.1111/j.1523-1739.1991.tb00370.x
MCCARTHY, M. A. 1998. Identifying declining and threatened species with museum data. Biol. Conservation. 83: 9-17. http://dx.doi.org/10.1016/S0006-3207(97)00048-7

MILlER, J. S., H. A. PORTER-MORGAN, H. STEVENS, B. BOOM, G. A. KRUPNICK, P. ACEVEDO-RODRIGUEZ, J. FLEMING \& M. GENSLER. 2012. Addressing target two of the Global Strategy for Plant Conservation by rapidly identifying plants at risk. Biodivers. \& Conservation 21: 1877-1887.

http://dx.doi.org/10.1007/s10531-012-0285-3

MILLER, J. S., G. A. KRUPNICK, H. STEVENS, H. PORTER-MORGAN, B. BOOM, P. ACEVEDORODRIGUEZ, J. ACKERMAN, D. KOLTERMAN, E. SANTIAGO, C. TORRES \& J. VELEZ. 2013. Toward target 2 of the global strategy for plant conservation: an expert analysis of the puertorican flora to validate new streamlined methods for assessing conservation status. Ann. Missouri Bot. Gard. 99: 199-205.

http://dx.doi.org/10.3417/2011121

NIC LUGHADHA, E., B. E. WALKER, C. CANTEIRO, H. CHADBURN, A. P. DAVIS, S. HARGREAVES, E. J. LUCAS, A. SCHUITEMAN, E. WILLIAMS, S. P. BACHMAN, D. BAINES, A. BARKER, A. P. BUDDEN, J. CARRETERO, J. J. CLARKSON, A. ROBERTS \& M. C. RIVERS. 2018. The use and misuse of herbarium specimens in evaluating plant extinction risks. Phil. Trans. R. Soc. B 37420170402. http://dx.doi.org/10.1098/rstb.2017.0402

NICHOLLS, N. 2004. The changing nature of Australian droughts. Climatic Change 63: 1473-1480. http:// dx.doi.org/ 10.1023/B:CLIM.0000018515.46344.6d

NOVOA, P. 2008. Gavilea cardioglossa. www.mma.gob. $\mathrm{cl} /$ clasificacionespecies/informacion-procesos-2014. htm

PARTOMIHARDJO, T. 2003. Colonisation of orchids on the Krakatau Islands. Telopea 10: 299-310.

PlanEAr 2009-2018. Plantas Endémicas de la Argentina. [online]. Disponible en: www.lista-planear.org [Acceso: 20 enero 2019].

PITMAN, N. C. A. \& JORGENSEN P. M. 2002. Estimating the Size of the World's Threatened Flora. Science 298: 989.

http://dx.doi.org/10.1126/science.298.5595.989

RECA, A., C. ÚBEDA \& D. GRIGERA. 1994. Conservación de la fauna de Tetrápodos I. Un índice para su evaluación. Mastozoología Neotropical 1: 17-28. 


\section{J. Dieta y F. Biganzoli - Evaluación preliminar del estado de conservación de plantas}

RIVERS, M. C., S. P. BACHMAN, T. R. MEAGHER, E. N. LUGHADHA \& N. A. BRUMMITT. 2010. Subpopulations, locations and fragmentation: applying IUCN red list criteria to herbarium specimen data. Biodivers. \& Conservation 19: 20712085. http://dx.doi.org/10.1007/s10531-010-9826-9

RODRÍGUEZ-ECHEVERRY, C. ECHEVERRÍA \& L. NAHUELHUAL. 2015. Impacts of anthropogenic land-use change on populations of the Endangered Patagonian cypress Fitzroya cupressoides in southern Chile: implications for its conservation. Oryx 49: 447-452.

http://dx.doi.org/10.1017/S0030605314000945

SALA, O. E., F. STUART CHAPIN III, J. J. ARMESTO, E. BERLOW, J. BLOOMFIELD, R. DIRZO, E. HUBER-SANWALD, L. F. HUENNEKE, R. B. JACKSON, A. KINZIG, R. LEEMANS, D. M. LODGE, H. A. MOONEY, M. OESTERHELD, N. LEROY POFF, M. T. SYKES, B. H. WALKER, M. WALKER \& D. H. WALL. 2000. Global biodiversity scenarios for the year 2100. Science 287: 1770-1774. http://dx.doi.org/10.1126/science.287.5459.1770

SCHATZ, G. E. 2009. Plants on the IUCN Red List: setting priorities to inform conservation. Trends $\mathrm{Pl}$. Sci. 14: 1360-1385.

http://dx.doi.org/10.1016/j.tplants.2009.08.012

STROPP, J., R. J. LADLE, A. C. M. MALHADO, J. HORTAL, J. GAFFURI, W. H. TEMPERLEY, J. O. SKØIEN \& P. MAYAUX. 2016. Mapping ignorance: 300 years of knowledge of flowering plants in Africa. Global Ecol. Biogeogr. 25: $1085-$ 1096. http://dx.doi.org/10.1111/geb.12468

STUART, S. N., WILSON E. O., MCNEELY J. A., MITTERMEIER R. A. \& RODRIGUEZ J. P. 2010. The Barometer of Life. Science 328: 177. http://dx.doi.org/ 10.1126/science.1188606

SWARTS, N. D. \& K. W. DIXON. 2009. Terrestrial orchid conservation in the age of extinction. Ann. Bot. 104: 543-556.

http://dx.doi.org/10.1093/aob/mcp025

SWINGLAND, I. R. 2001. Definition of Biodiversity. Encyclopedia of Biodiversity, Volume 1: 377-391.
TALA, CH. 2010. Gavilea kingii. [online]. Disponible en: www.mma.gob.cl/clasificacionespecies/ informacion-procesos-2014.htm [Acceso: 20 enero 2019].

THIERS, B., 2019. Index Herbariorum: A global directory of public herbaria and associated staff. New York Botanical Garden's Virtual Herbarium [online]. Disponible en: http://sweetgum.nybg.org/ science/ih/. (Acceso: 21.02.19).

UNEP 2002. United Nations Environment Programme. Global strategy for plant conservation. COP Decision VI/9, CBD Secretariat, Montreal Decision VI/9, CBD Secretariat, Montreal.

VANDEN BROECK, A., W. VAN LANDUYT, K. COX, L. DE BRUYN, R. GYSELINGS, G. OOSTERMEIJER, B. VALENTIN, G. BOZIC, B. DOLINAR, Z. ILYÉS \& J. MERGEAY. 2014. High levels off effective long-distance dispersal may blur ecotypic divergence in a rare terrestrial orchid. $B M C$ Ecology 14: 20. http://dx.doi.org/10.5061/dryad.sb68v.

VAN DYKE, F. 2008. Chapter 4. Biodiversity: Concept, Measurement, and Challenge. In: VAN DYKE, F. Conservation Biology: Foundations, Concepts, Applications. Springer. Pp: 83-119.

VIDAL, O. J., C. SAN MARTÍN, S. MARDONES, V. BAUK \& C. F. VIDAL. 2012. The orchids of Torres del Paine Biosphere Reserve: The need for species monitoring and ecotourism planning for biodiversity conservation. Gayana Botanica 69: 136-146.

VITOUSEK, P. M. 1992. Global Enviromental Change: An Introduction. Ann. Rev. Ecol. Syst. 23: 1-14. h t t p://dx.doi.org/10.1146/annurev. es.23.110192.000245

VOGEL ELY, C., S. A. L. BORDIGNON, R. TREVISAN \& I. I. BOLDRINI. 2017. Implications of poor taxonomy in conservation. J. Nat. Conservation 36: 10-13. http://dx.doi.org/10.1016/j.jnc.2017.01.003

ZULOAGA, F. O., O. MORRONE \& M. J. BELGRANO. 2008. Catálogo de Plantas Vasculares del Cono Sur. Monogr. Syst. Bot. Missouri Bot. Gard. 107: 1-3486. 
